\title{
Periodicity and chaos in electrically coupled Hindmarsh-Rose neurons
}

\author{
R. Erichsen, Jr., ${ }^{*}$ M. S. Mainieri, ${ }^{\dagger}$ and L. G. Brunnet ${ }^{\ddagger}$ \\ Instituto de Física, Universidade Federal do Rio Grande do Sul, Caixa Postal 15051, 91501-970 Porto Alegre, RS, Brazil
}

(Received 2 September 2005; revised manuscript received 1 November 2006; published 19 December 2006)

\begin{abstract}
The Hindmarsh-Rose (HR) system of equations is a model that captures the essential of the spiking activity of biological neurons. In this work we present an exploratory numerical study of the time activities of two HR neurons interacting through electrical synapses. The knowledge of this simple system is a first step towards the understanding of the cooperative behavior of large neural assemblies. Several periodic and chaotic attractors where identified, as the coupling strength is increased from zero until the perfect synchronization regime. In addition to the known phase locking synchronization at weak coupling, electrical synapses also allow for both in-phase and antiphase synchronization from moderate to strong coupling. A regime where the system changes apparently randomly between in-phase and antiphase locking evolves to a bistability regime, where both in-phase and antiphase periodic attractors are locally stable. At the strong coupling regime in-phase chaotic evolution dominates, but windows with complex periodic behavior are also present.
\end{abstract}

DOI: 10.1103/PhysRevE.74.061906

PACS number(s): 87.10.+e, 64.60.Cn, 07.05.Mh, 05.45.-a

\section{INTRODUCTION}

The Hindmarsh-Rose (HR) neuron model [1] can reproduce most of the different activity regimes of biological neurons. For different external input currents, the isolated HR neuron may stay quiescent, spike regular or chaotically, and burst regular or chaotically. See Ref. [2] for a detailed description of the single HR neuron behavior.

This work is concerned with the electrical coupling between two HR neurons. Electrical synapses, also called gap junctions are binary couplings between neurons where the current exchange is simply due to the difference between their membrane potential. Their existence in mammalian brain is nowadays well established. We refer to Ref. [3] for a detailed study of the role played by electrical synapses in promoting synchronization in a pair of integrate-and-fire model neurons, and the references therein for the evidence of the presence of electrical synapses in the mammalian brain.

The activity of coupled HR neurons is being largely studied in recent years. The switching among different phaselocking regimes, for small electrical coupling, was studied in Refs. [4,5]. Phase synchronization between chaotic neurons, electrically coupled, was studied in Refs. [6,7]. A detailed study of the synchronization between two coupled electronic HR neurons was presented in Ref. [8]. There, the authors show that excitatory coupling can produce in-phase burst synchronization, while antiphase burst synchronization results from inhibitory coupling. In the same paper, it was shown that an electronic HR neuron is able to synchronize in-phase with a living neuron of the lobster Panulirus interruptus. In Ref. [9] the the authors show that a twodimensional network in which each HR neuron is electrically coupled to its nearest neighbors shows bistability between a phase with global synchronization and a phase with partial synchronization. An accurate study of the phase synchronization state was presented in Ref. [10].

\footnotetext{
*Electronic address: rubem@if.ufrgs.br

†Electronic address: mainieri@if.ufrgs.br

*Electronic address: leon@if.ufrgs.br
}

The role of the coupling strength on the electrical activity of connected neurons was explored in a recent paper [11]. There, the authors noticed that with an increasing coupling strength, the incoherence is increased in a first moment, before decreasing to synchronized states by means of a double transition, being the first transition associated to synchronization of bursts and the second to the synchronization of spikes. See also Refs. [12,13].

We report here a numerical study of two electrically coupled HR neurons. Our goal was to explore the rich dynamical behavior that emerges from this system, mainly at moderate and strong coupling. To our knowledge, most of the chaotic and complex periodic attractors related to the two-neuron system were not yet discussed in the literature. In what follows we present our results and discussions concerning the moderate and strong coupling regime of chaotic HR neurons. At the end, we resume the paper and address some concluding remarks.

\section{THE HINDMARSH-ROSE MODEL}

The time evolution of a pair of HR neurons is described by a set of three differential equations for each neuron. With $i, j=1,2$, we have

$$
\begin{gathered}
\frac{d x_{i}}{d t}=y_{i}+3 x_{i}^{2}-x_{i}^{3}-z_{i}+I+\epsilon\left(x_{j}-x_{i}\right), \\
\frac{d y_{i}}{d t}=1-5 x_{i}^{2}-y_{i}, \\
\frac{d z_{i}}{d t}=-r z_{i}+r S\left(x_{i}+1.6\right) .
\end{gathered}
$$

The variable $x_{i}$ represents the membrane potential of the neuron labeled $i$. The variables $y_{i}$ and $z_{i}$ represent, respectively, "fast" and "slow" ion currents in the neuron dynamics. Constants $r, I$, and $S$ are model parameters representing currents and conductances, adjusted in order to reproduce the biologi- 


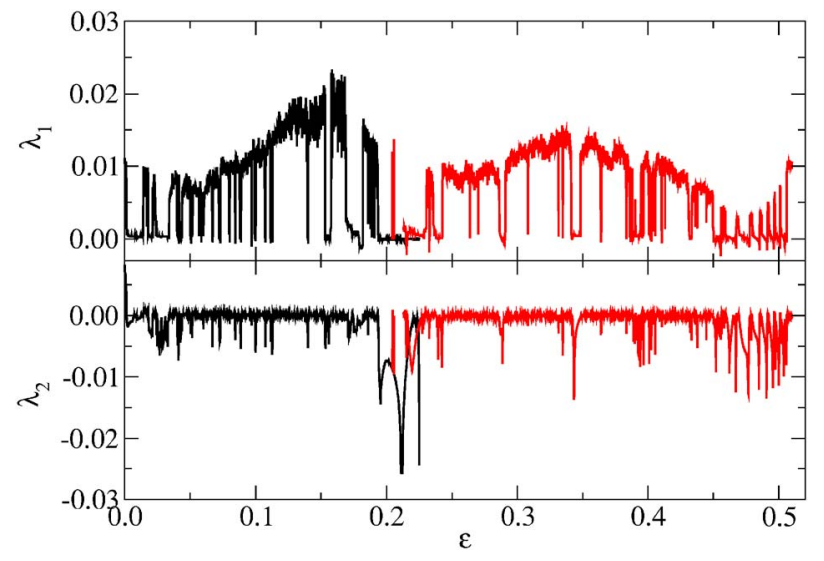

FIG. 1. (Color online) The largest and the second largest Lyapunov exponents as a function of the coupling $\epsilon$. Black line, small- $\epsilon$ attractor; grey (red online) line, large- $\epsilon$ attractor. The two attractors coexist in the interval $0.204 \lesssim \epsilon \lesssim 0.225$.

cal behavior. The electric coupling is introduced with the addition of an ohmic term to the first equation for each neuron. The parameter $\epsilon$ plays a role of a conductivity. Through all this work, we assume the literature values $r=0.0021$ and $S=4$ (see Ref. [9]). We fix the external current $I=3.38$, where the isolated neuron follows a chaotic evolution. The model equations were integrated using a variable step fourth-order Runge-Kutta algorithm.

\section{COUPLING OF CHAOTIC NEURONS}

A rich dynamical behavior is observed when two HR neurons are allowed to synchronize with a moderate to large coupling constant. We present in Fig. 1 the largest and the second largest Lyapunov exponents for $0 \leqslant \epsilon \leqslant 0.51$. The calculation of the Lyapunov spectrum was done by following the method presented in Ref. [14]. In the interval $0 \leqslant \epsilon \lesssim 0.0013$ the system remains as if uncoupled, with both neurons following a chaotic, uncorrelated evolution. In this interval, the Lyapunov spectra is twice degenerated. At $\epsilon \simeq 0.013$ the system synchronizes, and a 13 -spikes per burst periodic attractor becomes stable. The system enters into a phase-locking regime, but the phase differences cannot be calculated through a phase dynamic approach since the single neuron is not periodic.

Reaching $\epsilon \gtrsim 0.033$ chaotic evolution becomes dominating, but several windows of periodicity are observed. In general lines, for $\epsilon \lesssim 0.1936$ the chaotic evolution is as shown in Fig. 2, that corresponds to $\epsilon=0.15$. There is no pulse synchronization at all. Concerning the burst synchronization, when following a large time interval the system alternates

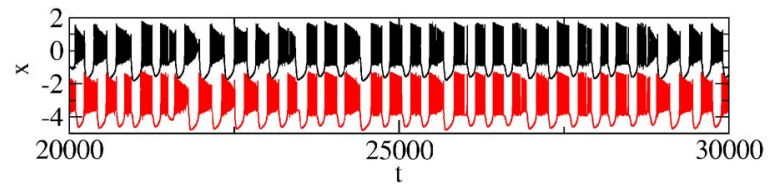

FIG. 2. (Color online) Chaotic time evolution of two neurons for $\epsilon=0.15$.
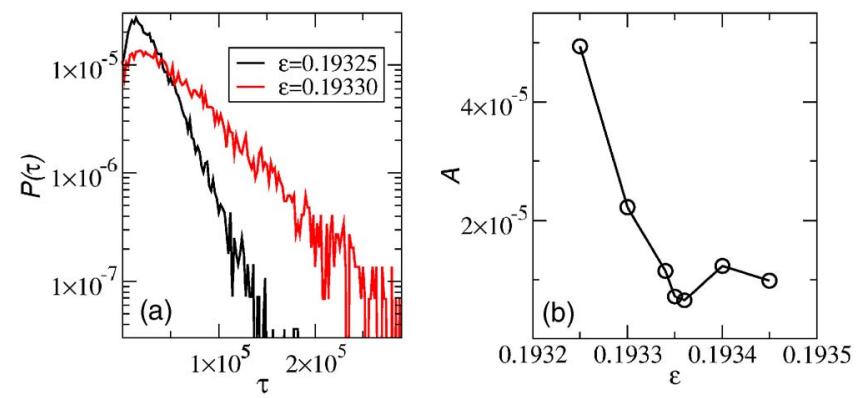

FIG. 3. (a) (Color online) Distribution of time intervals for a complete in-phase antiphase cycle for the chaotic evolution with $\epsilon<0.1936$. (b) Time constant $A$ of the exponential tail in $P(\tau) \sim \exp (-A \tau)$.

between in-phase and antiphase burst synchronization. It is also observed that as $\epsilon$ increases, the time interval for a complete in-phase or antiphase cycle increases, and seems to diverge for $\epsilon \rightarrow 0.1936$. In Fig. 3(a) the distribution of the time intervals is plotted in a log-linear plot, displaying an exponential tail. If an exponential law $P(\tau) \sim \exp (-A \tau)$ is adjusted to the tail, the relation between the time constant $A$ and the coupling $\epsilon$ is given by Fig. 3(b), showing a nonmonotonic decrease.

The picture that we can draw, for the increase of the time intervals, is that the chaotic attractor is composed of two distinct states in the phase space. As $\epsilon$ approaches 0.1936, it becomes more and more difficult the transit between these two states. The nonmonotonicity in the $A \times \epsilon$ relation means that the increase in the difficulty to pass from one part of the attractor to the other is not monotonic. This description lets us expect that for $\epsilon>0.1936$ the two attractors, the in-phase and the antiphase, should coexist. This is indeed the case. The two periodic attractors are shown in Fig. 4 for $\epsilon=0.205$. It is important to stress that the antiphase synchronization was obtained with the same electric coupling as the in-phase synchronization.

The coexistence of the two attractors is shown in the Lyapunov exponents (Fig. 1): in the interval $0.204 \lesssim \epsilon$ $\lesssim 0.225$ two pairs of exponents that are shown. The pair of exponents that correspond to the in-phase attractor is shown in grey (red online), while that corresponding to the antiphase is shown in black. It can be seen that the largest exponent for the in-phase solution is slightly positive. We are not sure if this positive value results from a lack of accuracy in our calculation, or if the in-phase attractor is marginally chaotic.

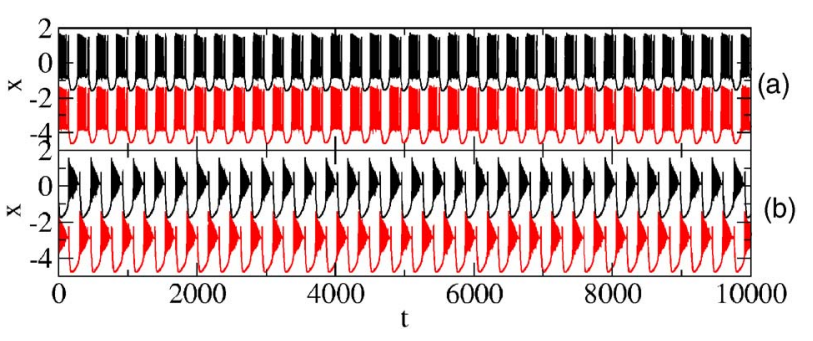

FIG. 4. (Color online) Coexisting (a) in-phase and (b) antiphase attractors for $\epsilon=0.205$. 


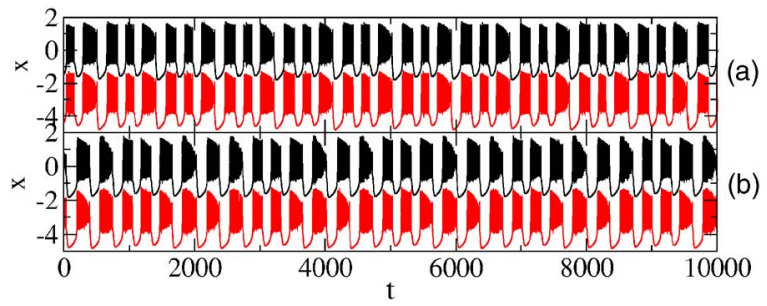

FIG. 5. (Color online) (a) six-burst long symmetric attractor for $\epsilon=0.27$. (b) six-burst long asymmetric attractor for $\epsilon=0.386$.

For $\epsilon \gtrsim 0.224$ only the in-phase attractor is stable. In the interval $0.23 \leqq \epsilon \lesssim 0.449$ the chaotic behavior with in-phase burst synchronization dominates, but several periodicity windows with very complexes periodic states can be found. Two examples are shown in Figs. 5(a) and 5(b), respectively, for $\epsilon=0.27$ and $\epsilon=0.386$. These examples can be used to introduce a classification to the periodic states. We define a bursting sequence of the form $n_{11} / n_{21}, n_{12} / n_{22}, \ldots, n_{1 l} / n_{2 k}$, where $n_{1 i} / n_{2 j}$ means a synchronized burst where neuron 1 spikes $n_{1 i}$ times while neuron 2 spikes $n_{2 j}$ times. For $\epsilon=0.27$ the periodic attractor has a bursting sequence of $23 / 24,16 / 15,15 / 14$. In the next three bursts, the two neurons change their role, completing the period with a bursting sequence of $24 / 23,15 / 16,14 / 15$. This means that the complete period of this state is six bursts long. Since the two neurons play the same role in the dynamics, we classify this as a symmetric state. The periodic attractor at $\epsilon=0.386$ shown in Fig. 5(b) has a bursting sequence of $23 / 23,22 / 23,16 / 16,17 / 17,22 / 22,24 / 25$. This is also a six bursts long period, but since the two neurons play a distinct role in the dynamics, we classify this as an asymmetric state.

Concerning the chaotic states, they consist of a sequence of bursts $n_{1 i} / n_{2 j}$, in general the same as those observed in the periodic states, but in a nonordered sequence.
We end this discussion by mentioning that in the interval $0.449 \leqq \epsilon \lesssim 0.51$ a periodic evolution dominates, with small chaotic intervals when the number of spikes per burst changes. At $\epsilon \sim 0.51$ the system becomes perfectly synchronized, behaving as a single neuron.

\section{CONCLUSIONS}

We have explored the rich dynamical behavior of a system composed of two chaotic neurons in the moderate to strong coupling regimes. At moderate coupling, chaotic alternation between in-phase and antiphase synchronization was observed. At the transition between moderate to strong coupling two periodic attractors, an in-phase and an antiphase one coexist. At the strong coupling regime, only inphase synchronization, with chaotic and complex periodic attractors can be observed.

We may question ourselves about the relevance of the fine details concerning the bursting dynamics, discussed above. Nowadays, it is under investigation what is important for the neural coding [15]. Is only the burst synchronization important, or are the individual pulses inside a burst also important? In this case, the study of fine details is justified, and we can advance that a working system should be tuned to operate in chaotic regime, where it can carry relevant information, contrary to the periodic regime. Further investigation should concentrate in measurements of the entropy of the two neurons system, and the robustness of the fine details to noise effects.

\section{ACKNOWLEDGMENTS}

This work was supported in part by the Brazilian agencies CNPq (Conselho Nacional de Desenvolvimento Científico e Tecnológico) e FAPERGS (Fundação de Amparo à Pesquisa do Estado do Rio Grande do Sul), Contract No. 0408751.
[1] J. L. Hindmarch and R. M. Rose, Proc. R. Soc. London, Ser. B 221, 87 (1984).

[2] X.-J. Wang, Physica D 62, 263 (1993).

[3] B. Pfeuty, G. Mato, D. Golomb, and D. Hansel, Neural Comput. 17, 633 (2005).

[4] S. H. Park, S. K. Han, S. Kim, C. S. Ryu, S. Kim, and T. Yim, ETRI J. 18, 161 (1996).

[5] S. H. Park, S. Kim, H.-B. Pyo, and S. Lee, Phys. Rev. E 60, 2177 (1999).

[6] J.-W. Shuai and D. M. Durand, Phys. Lett. A 264, 289 (1999).

[7] D. H. He, G. Hu, M. Zhan, and H. P. Lu, Physica D 156, 314 (2001).

[8] R. D. Pinto, P. Varona, A. R. Volkovskii, A. Szücs, H. D. I. Abarbanel, and M. I. Rabinovich, Phys. Rev. E 62, 2644
(2000).

[9] R. Huerta, M. Bhazenov, and M. I. Rabinovich, Europhys. Lett. 43, 719 (1998).

[10] M. S. Mainieri, R. Erichsen, Jr., and L. G. Brunnet, Physica A 354, 663 (2005).

[11] M. Dhamala, V. K. Jirsa, and M. Ding, Phys. Rev. Lett. 92, 028101 (2004).

[12] Y. Jiang, Phys. Rev. Lett. 93, 229801 (2004).

[13] M. Dhamala, V. K. Jirsa, and M. Ding, Phys. Rev. Lett. 93, 229802 (2004).

[14] J.-P. Eckmann and D. Ruelle, Rev. Mod. Phys. 57, 617 (1985).

[15] M. I. Rabinovich, R. D. Pinto, H. D. I. Abarbanel, E. Tummer, G. Stiesberg, R. Huerta, and A. Selverston, Network Comput. Neural Syst. 13, 487 (2002). 